\title{
Evaluation of synergistic osteogenesis between icariin and BMP2 through a micro/meso hierarchical porous delivery system
}

This article was published in the following Dove Press journal:

International Journal of Nanomedicine

19 October 2017

Number of times this article has been viewed

\author{
Qian Wang ${ }^{1-4}$ \\ Lingyan $\mathrm{Cao}^{4,5}$ \\ Yang Liu ${ }^{6}$ \\ Ao Zheng 4,5 \\ Jiannan $\mathrm{Wu}^{4,5}$ \\ Xinquan Jiang ${ }^{4,5}$ \\ Ping $\mathrm{Ji}^{1-3}$
}

'Department of Oral and Maxillofacial Surgery, Stomatological Hospital of Chongqing Medical University, ${ }^{2}$ Chongqing Key Laboratory of Oral

Diseases and Biomedical Sciences, ${ }^{3}$ Chongqing Municipal Key Laboratory of Oral Biomedical Engineering of Higher Education, Chongqing, ${ }^{4}$ Oral Bioengineering and Regenerative Medicine Lab, Shanghai Key Laboratory of Stomatology \& Shanghai Research Institute of Stomatology, National Clinical Research Center of Stomatology, ${ }^{5}$ Department of Prosthodontics, Ninth People's Hospital, Shanghai Jiao Tong University School of Medicine, ${ }^{6}$ The State Key Laboratory of Bioreactor Engineering, East China University of Science and Technology, Shanghai, People's Republic of China
Correspondence: Xinquan Jiang; Ping Ji Department of Oral and Maxillofacial Surgery, Stomatological Hospital of Chongqing Medical University, 426 Jinlong Road, Chongqing 402160, People's Republic of China Email xinquanjiang@aliyun.com; jiping_62@hotmail.com
Abstract: BMP2 is well known as an outstanding growth factor for inducing new bone formation. However, improvements are still required to use BMP2 effectively and expand its clinical application due to the potential side effects at high doses. In this study, icariin (IC), a type of traditional Chinese medicine, was originally proposed to be a cooperative factor for BMP2. An alkaline phosphatase (ALP) activity assay showed that IC promoted BMP2 osteogenesis in a concentration-dependent manner with significant enhancement at $38.4 \mu \mathrm{M}$ versus that for BMP2 at $0.8 \mu \mathrm{g} / \mathrm{mL}$. Furthermore, we developed a composite hierarchical porous scaffold (SF/SBA15; composed of micropores of silk fibroin [SF] scaffold and mesopores of SBA15) for the controlled delivery of BMP2 and IC. This composite scaffold was investigated by a series of physical characterizations and displayed good in vitro cell biocompatibility. In addition, the composite scaffold also showed the degradation rate of $12 \%$ dry weight loss and a slight change in the microstructures within 10 days. Moreover, BMP2 and IC were loaded into the SF and SBA15 structures, respectively, of the SF/SBA15 scaffold. This protein/drug loading system $\left(\mathrm{SF}_{\mathrm{BMP} 2} / \mathrm{SBA} 15_{\mathrm{IC}}\right)$ provided delivery of BMP2 with an initial burst release of $60.9 \% \pm 0.9 \%$ in the first 24 hours and a gradual release over the subsequent 6 days to $97.9 \% \pm 0.8 \%$, whereas IC exhibited a burst release of $64.2 \% \pm 0.7 \%$ in the first 24 hours, followed by a sustained release to $92.4 \% \pm 0.8 \%$ over 10 days. With the prolonged local retention and interaction duration of $\mathrm{BMP} 2$ and IC, the $\mathrm{SF}_{\mathrm{BMP} 2} / \mathrm{SBA} 15_{\mathrm{IC}}$ scaffold provided better osteogenic differentiation than other groups with different loading modes of BMP2 or IC, as determined by ALP staining and quantitation and Alizarin red staining. Finally, the results of quantitative real-time polymerase chain reaction analysis indicated that the $\mathrm{SF}_{\mathrm{BMP} 2} / \mathrm{SBA} 15_{\mathrm{IC}}$ scaffold induced a significantly higher increase in the RUNX2, $A L P, C O L I$, and $O C N$ expression levels of cocultured bone marrow mesenchymal stem cells than other payload composite scaffolds. This study suggests that a micro/meso hierarchical porous delivery system of BMP2 and IC ensures osteogenic synergy and demonstrates promise for bone tissue engineering.

Keywords: synergistic delivery, hierarchical structure, controlled release, porous scaffold, mesopore, growth factor

\section{Introduction}

Bone defects, especially large-volume defects, often arise from trauma, skeletal diseases, congenital malformations, and tumor resections ${ }^{1}$ and remain challenging to surgeons. Traditional approaches for bone reconstruction include autologous bone grafting and alloplastic implants. However, the risks of limited supply, secondary traumatization, disease transmission, and immunogenicity still exist. ${ }^{2,3}$ The limitations of autografts and allografts have compelled the development of artificial materials that can stimulate 
natural bone tissue formation and repair. ${ }^{4}$ Tissue engineering approaches for bone regeneration based on the potent osteoinductive activity of BMPs have been proposed. Among BMPs, BMP2 has exhibited great potential for the regeneration of bone defects due to its remarkable ability to gather osteoprogenitor cells and induce osteogenic differentiation. ${ }^{4}$

To efficiently promote bone healing, the application of BMP2 has been widely used and is approved by the US Food and Drug Administration. ${ }^{4,5}$ However, despite its clinical efficacy in bone regeneration, BMP2 is limited by its short duration of bioactivity, highly preserved condition, and high production costs. Furthermore, at high doses, BMP2 is associated with risks and side effects, such as excessive bone resorption ${ }^{6}$ and the promotion of tumor angiogenesis. ${ }^{7,8}$ Therefore, it is necessary to find effective biomolecules that can synergistically promote the osteogenic bioactivity of BMP2. Osteogenic bioactivity can also be enhanced through biochemical cues via the combination of effective biomolecules, such as heparin, ${ }^{9} 2-\mathrm{N}, 6-\mathrm{O}$-sulfated chitosan, ${ }^{10}$ dexamethasone, ${ }^{3}$ and vascular endothelial growth factor. ${ }^{4,11}$ Traditional Chinese medicines characterized by high production rates and low cost could be used in the synergy with BMP2. However, there are few studies regarding traditional Chinese medicines that may coordinate with BMP2. Among the traditional Chinese medicines, icariin (IC), which is extracted from Epimedium, is a common drug that has been used to treat bone fractures and osteoporosis for thousands of years in People's Republic of China. ${ }^{12-15}$ In our previous study, we demonstrated that IC promoted the osteogenic differentiation and expression of angiogenic factors of bone marrow mesenchymal stem cells (BMSCs) and that a concentration of $20 \mu \mathrm{M}$ had the strongest in vitro stimulatory effect. ${ }^{16,17}$ Hence, synergy may exist between IC and BMP2, and it is possible that the BMP2 dosage could be reduced in the presence of IC.

Another possible effective method is to utilize a controlled delivery system that can facilitate osteogenesis with a low dosage of BMP2. IC also requires a suitable controlled delivery system to guarantee the release of the effective concentration that synergistically promotes the osteogenic bioactivity of BMP2. For the repair of critical-sized bone defects, utilizing biodegradable porous scaffolds that ensure the transportation of nutrients and promote the osteogenesis of bone regeneration is ideal. ${ }^{7,10}$ Consequently, a biodegradable porous scaffold as a delivery system that not only loads BMP2 and IC but also provides suitable delivery of the optimum concentrations and promotes local retention of the growth factor or drug on-site is required. Biocompatibility is the most important characteristic in designing an ideal scaffold for bone regeneration. The selected material and its degradation by-product should be biocompatible and noncytotoxic. Porous scaffolds have been fabricated using a wide range of materials including metals, ceramics, glass, polymers, composites, and their combinations. Among them, silk fibroin (SF), a natural macromolecular protein polymer ${ }^{18}$ with outstanding biocompatibility, biodegradability, and low inflammatory response, has been explored for use in various tissue engineering applications. ${ }^{4}$ In addition, ordered mesoporous materials (OMMs) possess a network of channels and pores of well-defined size in the nanoscale range $(2-50 \mathrm{~nm})$. This particular pore architecture makes them suitable candidates for hosting and delivering a variety of molecules under the appropriate conditions. ${ }^{19}$ Thereamong, SBA15 as a major breakthrough in polymer-templated OMMs showed excellent features including large surface areas, tunable nanometer-scale pore sizes, well-defined surface properties, and high hydrothermal stability. ${ }^{20-23}$ Moreover, they also possess abundant surface silanol groups that can be easily functionalized by organic active components. ${ }^{24}$ Therefore, as a porous nanocarrier, SBA15 is a good candidate with a high surface area and large pore volume, allowing high loading capacity.

To further enhance and control the delivery of BMP2 and IC, specific carriers have been identified that enhance bioactivity. ${ }^{25}$ In the present study, we prepared IC-loaded SBA15 nanoparticles and composite hydrogels of SF incorporating BMP2. We investigated the physical properties and biocompatibility of the scaffold as an effective controlled delivery vehicle for in vitro osteogenesis. In this study, a new delivery system with a micro/meso hierarchical porous structure was developed for controlling the dual delivery of BMP2 and IC to promote highly efficient osteogenesis (Figure 1). Controlled dual delivery of the two model bioactive molecules can be achieved by using this SBA15 nanoparticle-derived SF scaffold. The osteogenic differentiation and osteogenesis of this delivery system were further evaluated.

\section{Materials and methods BMSC isolation and culture}

This process was performed as previously described. ${ }^{16}$ Primary BMSCs were harvested from Sprague-Dawley rats (Shanghai SLAC Experimental Animal Center, Shanghai, People's Republic of China). All experimental protocols regarding the use of animals in this study were approved by the Institutional Animal Care and Use Committee and followed the procedure for Animal Experimental Ethical Inspection of 


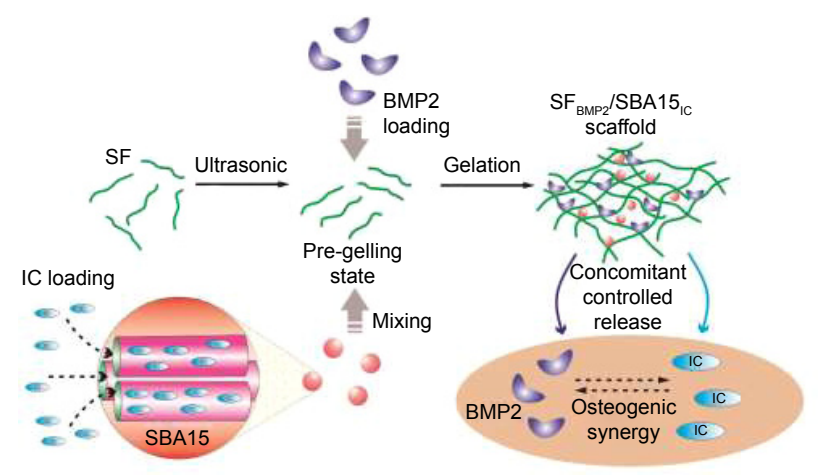

Figure I Schematic diagram of preparation of the micro/meso hierarchical porous delivery system for the performance of interaction between BMP2 and icariin. After the ultrasonic treatment, SF solution was well mixed with BMP2 and IC-loaded SBA I5. Consequently, the composite solution rapidly formed the hydrogel based on the conformational transitions of SF molecules and then lyophilized to generate the $\mathrm{SF}_{\mathrm{BMP} 2} / \mathrm{SBA} 15_{\mathrm{IC}}$ scaffold. This composite scaffold was designed to provide matched release profiles respectively for BMP2 and icariin, to guarantee their local retention time and the possible osteogenic synergy between them.

Abbreviations: IC, icariin; SF, silk fibroin.

the Ninth People's Hospital, which is affiliated with Shanghai Jiao Tong University School of Medicine. Bone marrow was flushed out with DMEM (HyClone, Logan, UT, USA) supplemented with $1 \%$ penicillin/streptomycin (HyClone). After blood cells were removed by centrifugation, the collection was mixed with complete DMEM supplemented with $10 \%$ fetal bovine serum (FBS; HyClone) and plated into a tissue culture flask. When large colonies formed and became confluent, the cells were trypsinized and passaged. BMSCs acquired from Passages 2 to 3 were used for our experiments.

\section{Synergy of IC and BMP2}

Rat BMSCs were seeded into 96-well tissue culture plates (TCPs) at a density of $5 \times 10^{3}$ cells/well, cultured with $0.8 \mu \mathrm{g} / \mathrm{mL}$ BMP2 and various concentrations (ie, $0,1.2,2.4$, $4.8,9.6,19.2,38.4,76.8$, and $156.3 \mu \mathrm{M}$ ) of IC. An alkaline phosphatase (ALP) activity quantitative assay of BMSCs was performed at 3 and 7 days. Samples from all groups were incubated with para-nitrophenyl phosphate (Sigma-Aldrich, St Louis, MO, USA) at room temperature for 60 minutes. Absorbance values (OD) were recorded at $405 \mathrm{~nm}$ to evaluate the ALP activity. The total protein content was assessed by using a BCA protein assay kit (Sigma-Aldrich), and the OD values were normalized to the bovine serum albumin (Sigma-Aldrich) standard curve at $590 \mathrm{~nm}$. The relative ALP activity of each individual counterpart was assessed as an OD value at $405 \mathrm{~nm}$ per milligram of total protein and then normalized to the fold changes using the control group without the presence of IC as the reference value (set to 1) and expressed as mean \pm SD. All the experiments were performed in triplicate.

\section{Synthesis of the hierarchical porous delivery system \\ Preparation of SBAI5}

SBA15 materials were synthesized according to the procedures described by Zhao et al. ${ }^{26}$ Briefly, $8 \mathrm{~g}$ of triblock copolymer Pluronic P123 (BASF, Antwerp, Belgium) was dissolved in $240 \mathrm{~mL}$ of $2 \mathrm{M} \mathrm{HCl}$ solution. This mixture was placed under magnetic stirring at $40^{\circ} \mathrm{C}$ until transparent micelles formed. Then, $17 \mathrm{~g}$ of tetraethyl orthosilane (Acros Organics, Geel, Belgium) was added under stirring. After 24 hours, the suspension was placed in a high-pressure reactor for 48 hours at $110^{\circ} \mathrm{C}$. Finally, the synthesized products were washed with demineralized water three times, dried, and calcined at $600^{\circ} \mathrm{C}$ with a heating rate of $1{ }^{\circ} \mathrm{C} / \mathrm{min}$ for 6 hours in a muffle furnace to obtain SBA15 powder. ${ }^{27}$

\section{Preparation of SF solution}

SF solution was prepared as previously described. ${ }^{28,29}$ Briefly, Bombyx mori silkworm cocoons were cut into small pieces and boiled for 30 minutes in a solution of $0.5 \mathrm{wt} \% \mathrm{Na}_{2} \mathrm{CO}_{3}$. After three rinses with ultrapure water, the extracted fibroin fibers were then dried at $60^{\circ} \mathrm{C}$ for 6 hours. The dried SF was dissolved in a triad solvent of $\mathrm{CaCl}_{2} / \mathrm{H}_{2} \mathrm{O} / \mathrm{EtOH}$ at a mole ratio of $1: 8: 2$ at $100^{\circ} \mathrm{C}$ and stirred for $\sim 10$ minutes until fully dissolved. After centrifugation to remove impurities, the solution was dialyzed against distilled water for 2 days by using dialysis cassettes (molecular weight cutoff $=3,500$; Pierce, Waltham, MA, USA) and then added to a $10 \mathrm{wt} \%$ poly(ethylene glycol) solution. The SF solution was sterilefiltered through a $0.22-\mu \mathrm{m}$ membrane and lyophilized for storage until needed for experiments. In our previous experiment, we found that the lyophilized SF could be redissolved in water, and when sonicated at the conditions of $50 \%$ amplitude and $20 \mathrm{kHz}$ for 3 minutes, the SF solution would gelatinize again at $60^{\circ} \mathrm{C}$ in 15 minutes.

\section{Preparation of the hierarchical porous delivery system $\left(\mathrm{SF}_{\text {BMP2 }} / \mathrm{SBA}\right.$ I $5_{\text {IC }}$ group)}

The designed system was established by loading mesoporous SBA15 carrying IC into microporous SF scaffolds carrying BMP2 to form a hierarchical architecture that could be used as a biomolecular delivery system and could simultaneously strengthen the synergistic action of biomolecules. Because of its much larger dimensions, BMP2 cannot be loaded into mesoporous SBA15. ${ }^{30,31}$ As a result, there was no group in which BMP2 was loaded into mesoporous SBA15.

To prepare the $\mathrm{SF}_{\mathrm{BMP} 2} / \mathrm{SBA} 15_{\text {IC }}$ delivery system, IC-loaded SBA15 was first prepared. Briefly, $67.7 \mathrm{mg}$ of IC (Tauto 
Biotechnology Company, Shanghai, People's Republic of China) was dissolved in $10 \mathrm{~mL}$ of dimethyl sulfoxide to form a $10 \mathrm{mM}$ stock solution. For each sample, both $3.3 \mathrm{mg}$ of SBA15 and $18 \mu \mathrm{L}$ of IC stock solution were placed in a tube for absorption overnight. Then, the whole tube was treated with the lyophilization to obtain the IC-loaded SBA15. For each sample, the lyophilized SF (32 mg) was dissolved in $400 \mu \mathrm{L}$ of PBS to yield an $8 \mathrm{wt} \%$ SF solution and then sonicated at $50 \%$ amplitude and $20 \mathrm{kHz}$ for 3 minutes. Then, $8 \mu \mathrm{g}$ of BMP2 (Rebone Biomaterials Co. Ltd., Shanghai, People's Republic of China) was added to the sonicated SF solution with vortex oscillation. Subsequently, the mixture BMP2/SF was transferred to the abovementioned tube containing the IC-loaded SBA15 to form the composite pre-gel. Finally, the prepared pre-gel solution was cast into a Teflon ${ }^{\circledR}$ mold (diameter $=10 \mathrm{~mm}$; height $=8 \mathrm{~mm}$ ) at room temperature for gelatinization and lyophilization to form the hierarchical architectural scaffold. During the process of fabricating the specific scaffold, four control groups were included: 1) a group with no loading of BMP2 or IC (SF/SBA15); 2) a group with the pure mesoporous SBA15 loaded into the microporous SF scaffold carrying BMP2 ( $\left.\left.\mathrm{SF}_{\mathrm{BMP} 2} / \mathrm{SBA} 15\right) ; 3\right)$ a group with the mesoporous SBA15 carrying IC loaded into the pure microporous SF (SF/SBA15 ${ }_{\mathrm{IC}}$ ); and 4) a group with the pure mesoporous SBA15 loaded into the microporous SF scaffold carrying both BMP2 and IC ( $\left.\mathrm{SF}_{\mathrm{BMP} 2 / \mathrm{IC}} / \mathrm{SBA} 15\right)$. All preparation procedures were performed in a sterile environment.

\section{Physical characterization of the SF/SBAI5 scaffold}

The morphology of the delivery system was studied by using a field emission scanning electron microscope (FE-SEM; S4800; Hitachi, Tokyo, Japan) with an accelerating voltage of $10 \mathrm{kV}$ via gold sputter coating. The elements on the scaffold surface were analyzed via energy-dispersive X-ray spectrometry (EDS-XRS; QUANTAX 400-30, BRUKER, Karlsruhe, Germany). High-resolution transmission electron microscopy (TEM; JEM-2100; JEOL, Tokyo, Japan) with a voltage of $200 \mathrm{kV}$ was used to characterize the morphology and microstructure of SBA15. The Brunauer-Emmett-Teller (BET) method was used to measure the surface area, the nanopore size distribution, and the pore volume via nitrogen adsorption-desorption isotherms based on Barrett-JoynerHalenda (BJH) analyses by using an analyzer (NOVA 4200e; Quantachrome, Boynton Beach, FL, USA).

\section{In vitro degradation study}

The degradation of the composite scaffold was studied. Each lyophilized composite scaffold SF/SBA15 (diameter $=1 \mathrm{~cm}$, height $=5 \mathrm{~mm})$ was immersed in $10 \mathrm{~mL}$ PBS $(\mathrm{pH}=7.4)$ and shaken at $100 \mathrm{rpm}$ and $37^{\circ} \mathrm{C}$. At designated time points (ie, 6 and 12 hours; 1 day; 2, 3, 4, 5, 6, 7, and 10 days), the samples were collected and lyophilized and then weighted respectively. The initial weight of the scaffold was noted $\left(\mathrm{W}_{\mathrm{i}}\right)$. The dried weight at designated time points was noted $\left(\mathrm{W}_{t}\right)$. Equation 1 was used to calculate the rate of degradation.

Degradation (rate of weight loss $\%)=\frac{\mathrm{W}_{\mathrm{i}}-\mathrm{W}_{\mathrm{t}}}{\mathrm{W}_{\mathrm{i}}} \times 100 \%$ (1)

The degradation rate was recorded as the mean $\pm \mathrm{SD}$ $(n=3)$. In addition, the samples at Day 10 were analyzed via FE-SEM, EDS-XRS, BET, and TEM to evaluate the morphology and microstructure of the composite scaffold and SBA15. Before characterization, the composite scaffolds were lyophilized and grinded first.

\section{In vitro release kinetics of BMP2 and IC}

The release kinetics of BMP2 and IC loaded into the different types of scaffolds were examined over a period of 7 days and 10 days, respectively. The scaffolds were placed in test tubes and immersed in $5 \mathrm{~mL}$ of $\mathrm{PBS}(\mathrm{pH}=7.4)$ at $37^{\circ} \mathrm{C}$ under constant agitation at $100 \mathrm{rpm}$. The buffer was collected $(1 \mathrm{~mL})$ at different incubation times, frozen, and stored at $-80^{\circ} \mathrm{C}$. The collected release buffer was replenished using an equal volume of fresh PBS. At the end of the experiment, all the collected samples were thawed and quantitatively analyzed for contents of BMP2 and IC. BMP2 content was determined by using a Human BMP2 ELISA Kit (Sigma-Aldrich), whereas IC was quantified by using a high-performance liquid chromatography system (Agilent 1260SL; Agilent, Santa Clara, CA, USA). Three samples were tested for each group at each time point, and the results were reported as mean $\pm \mathrm{SD}$. The cumulative release is expressed as the concentration in the medium as well as the percentage of total loaded protein or drug.

\section{Cell biocompatibility}

Rat BMSCs were seeded at a density of $1 \times 10^{5}$ per SF/SBA15 scaffolds, attached overnight in DMEM with 10\% FBS containing $100 \mathrm{U} / \mathrm{mL}$ of penicillin and $100 \mathrm{U} / \mathrm{mL}$ of streptomycin at $37^{\circ} \mathrm{C}$ in $5 \% \mathrm{CO}_{2}$ in a humidified environment. A 24-well TCP seeded with a density of $1 \times 10^{5}$ cells/well with no scaffold was used as a control group. The culture medium was replaced every 3 days. The cell viability of BMSCs was evaluated after 24 hours via a live/dead assay kit (Abcam, Cambridge, UK) according to the manufacturer's 
protocol. In brief, the cells in the scaffold were washed with sterile PBS, then incubated with standard working reagents for 25 minutes at $37^{\circ} \mathrm{C}$, and finally observed by confocal laser scanning microscopy (CLSM) (Nikon A1R; Nikon, Tokyo, Japan).

The cell morphology and alignment were studied by staining the filamentous actin of the cytoskeleton. After 24 hours of incubation in the composite SF/SBA15 scaffold, the cells on the surface of the sample were rinsed three times with PBS and fixed with 4\% paraformaldehyde for 15 minutes. After washing, the cells were incubated with fluorescein isothiocyanate-labeled phalloidin (SigmaAldrich) to stain cytoskeletal F-actin fibers. The sample was then washed again with PBS, and the cell nuclei were stained with 4',6-diamidino-2-phenylindole (Sigma-Aldrich). The cell morphology and alignment were visualized by using CLSM.

In addition, cell biocompatibility was assessed by using a tetrazolium dye (MTS) assay according to the manufacturer's instructions. After 1, 3, 5, and 7 days, the culture medium was replaced with fresh medium containing MTS according to the manufacturer's instructions. After 1.5 hours of incubation at $37^{\circ} \mathrm{C}$, the absorbance of the solution was measured at $490 \mathrm{~nm}$ (ELX-800; BIO-TEK, Winooski, VT, USA). The cell viability was calculated by normalizing the absorbance of the samples at $490 \mathrm{~nm}$ to that of the control. All samples were tested in triplicate, and the cell viability was expressed as the mean $\pm \mathrm{SD}$.

\section{In vitro mineralization}

Rat BMSCs were seeded into 12-well TCPs at a density of $2 \times 10^{5}$ cells/well, followed by coculture with a transwell system (Corning, New York, NY, USA) loaded with the five groups of scaffolds. On Day 7, each plate was rinsed three times with PBS and fixed with 4\% paraformaldehyde for 15 minutes. After being washed twice with distilled water, the cells were subjected to ALP staining (Beyotime, Shanghai, People's Republic of China) according to the manufacture's instruction. Stained cells were examined under a microscope (Eclipse Ti; Nikon). An ALP activity quantitative assay was also performed at the same time, exactly as in the described assay for the IC and BMP2 synergy. The relative ALP activity of each individual counterpart was assessed as an OD value at $405 \mathrm{~nm}$ per milligram of total protein and then normalized to the fold changes by using the control group SF/SBA15 as the reference value (set to 1 ) and expressed as the mean \pm SD. Half of the culture medium was replaced at 3 days. In addition, on Days 14 and 28, fixed cells were stained with 1\% Alizarin red (Sigma-Aldrich) for 10 minutes. The cells were then rinsed with water and viewed with an inverted light microscope (Eclipse Ti; Nikon). Half of the culture medium was replaced at $3,7,10,14,17,21$, and 24 days.

\section{Quantitative real-time polymerase chain reaction (PCR) assay}

Rat BMSCs were plated into 12-well plates at a density of $2 \times 10^{5}$ cells/well and incubated for 24 hours, followed by coculture with a transwell system loaded with the five groups of scaffolds. Cells cultured in 12-well TCPs without scaffolds served as a blank control. Total RNA was isolated from BMSCs at Days 3 and 7. At each time point, total RNA was isolated with TRIzol reagent (Life Technologies, Carlsbad, CA, USA) according to the manufacturer's instructions. The RNA concentrations were determined by using a NanoDrop spectrophotometer (ND-1000; Thermo, Wilmington, DE, USA). cDNA was synthesized via a cDNA synthesis reverse transcription kit (Takara, Tokyo, Japan). Then, cDNA synthesis was performed by using a Prime Script First-Strand cDNA Synthesis kit (Takara). Quantitative real-time PCR analysis was performed by using a ROCHE480 real-time PCR system (LightCycler480, Roche, Rotkreuz, Switzerland) for osteogenic markers of RUNX2, ALP, COL I, and OCN. $\beta$-Actin was used as an internal control. The data were analyzed by using the comparative $\mathrm{Ct}\left(2^{-\Delta \Delta \mathrm{Ct}}\right)$ method and are expressed as fold changes with respect to the control. The primer sequences used in the present study were synthesized commercially (Shengong, Co. Ltd., Shanghai, People's Republic of China), and Table 1 lists the specific primer sets. All the experiments were performed in triplicate. The analysis was based on calculating the relative expression levels of bone marker genes compared with the expression of controls on Days 3 and 7, and all values were normalized to $\beta$-Actin.

\section{Statistical analysis}

All quantitative data are expressed as the mean \pm SD and were analyzed with Origin 8.0 (OriginLab Corporation,

Table I List of primers used and the respective forward and reverse sequences

\begin{tabular}{ll}
\hline Gene & Forward and reverse sequences \\
\hline Runx2-F & 5'-ATCCAGCCACCTTCACTTACACC-3' \\
Runx2-R & 5'-GGGACCATTGGGAACTGATAGG-3' \\
OCN-F & 5'-GCCCTGACTGCATTCTGCCTCT-3' \\
OCN-R & 5'-TCACCACCTTACTGCCCTCCTG-3' \\
ALP-F & 5'-TATGTCTGGAACCGCACTGAAC-3' \\
ALP-R & 5'-CACTAGCAAGAAGAAGCCTTTGG-3' \\
Col I-F & 5'-CAGGCTGGTGTGATGGGATT-3' \\
Col I-R & 5'-CCAAGGTCTCCAGGAACACC-3' \\
$\beta$ B-Actin-F & 5'-GTAAAGACCTCTATGCCAACA-3' \\
$\beta$ B-Actin-R & 5'-GGACTCATCGTACTCCTGCT-3' \\
\hline
\end{tabular}




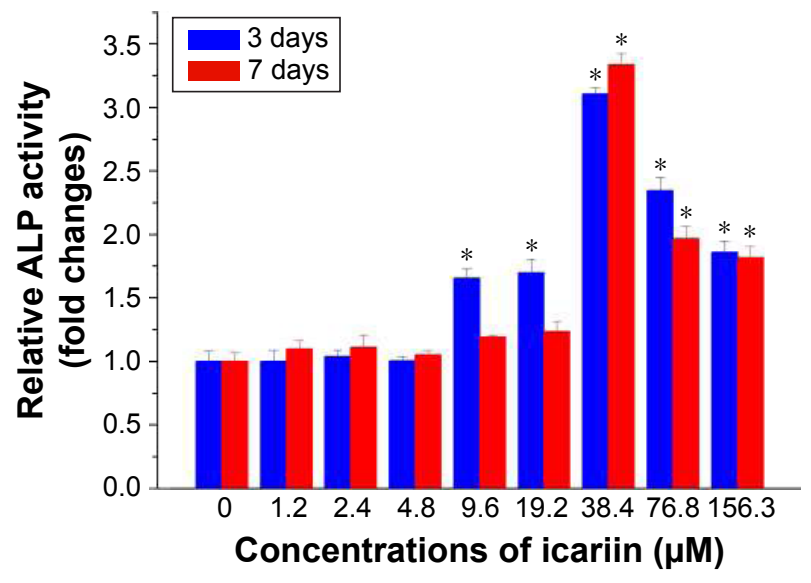

Figure 2 Osteogenic synergy of icariin and BMP2 measured by ALP activity quantitative assay. Rat BMSCs were treated in advance with $0.8 \mu \mathrm{g} / \mathrm{mL} \mathrm{BMP2}$ and different concentrations of icariin before 3 days and 7 days. ${ }^{*} p<0.05$ represents a significant difference to the group without the presence of icariin at the corresponding time point.

Abbreviations: ALP, alkaline phosphatase; BMSC, bone marrow mesenchymal stem cell.

Northampton, MA, USA). Statistical comparisons were carried out by using analysis of variance. Confidence levels $>95 \%$ $(p<0.05)$ were considered statistically significant.

\section{Results and discussion Synergy of IC with BMP2}

The relative ALP activities for the synergy between different concentrations of IC and BMP2 were analyzed at Days 3 and 7 (Figure 2). Cells incubated with different densities of IC and BMP2 showed different levels of ALP activity, demonstrating the synergy of their osteogenesis effects. The medium containing IC at concentrations from 9.6 to $156.3 \mu \mathrm{M}$ at 3 days and from 38.4 to $156.3 \mu \mathrm{M}$ at 7 days resulted in a significantly synergistic increase in ALP activity induced by $0.8 \mu \mathrm{g} / \mathrm{mL}$ BMP2. The ALP activity exhibited an increasing trend with increasing IC concentrations from 0 to $38.4 \mu \mathrm{M}$, followed by a decreasing trend when IC was further increased from 38.4 to $156.3 \mu \mathrm{M}$. The strongest ALP activity was observed for $38.4 \mu \mathrm{M}$ IC. This phenomenon is possibly due to the cytotoxicity of IC at high concentrations.
In our previous study, ${ }^{17} \mathrm{IC}$ was evidenced to have no enhancement on cell proliferation by cell cycle detection. MTS assay showed that IC at a concentration of $>40 \mu \mathrm{M}$ had a certain degree of cytotoxicity.

\section{Physical properties}

The morphology of the composite scaffold imaged by SEM reveals the structures of the SF scaffold loaded with SBA15 (Figure 3). The SF scaffold had a net-like microstructure with a diameter of $\sim 100 \mu \mathrm{m}$ and contained evenly and regularly distributed SBA15 nanoparticles, which were present on the walls of the pores. Notably, the SF/SBA15 scaffold had many nanoparticles that provided a hydrophilic layer at the surface for better cell attachment and spreading.

EDS-XRS mapping analysis revealed the elemental distributions of carbon, nitrogen, and oxygen, which are representatives of SF, and of silicon, which is representative of SBA15, on the scaffold surfaces (Figure 4). The images show that the silicon was evenly and widely distributed on the scaffold surface. The distribution of silicon was significantly higher on the SF scaffold surface, suggesting that excellent biomineralization may be induced on the scaffold with SBA15. The elemental analyses of the SF/SBA15 scaffold showed that the carbon, oxygen, nitrogen, and silicon contents were $50.95 \%, 30.12 \%, 16.61 \%$, and $2.33 \%$, respectively.

Figure 5 presents the characterization of SBA15. It is well known that SBA15 comprises tubular mesopores in an ordered array. ${ }^{26}$ Low-temperature nitrogen adsorptiondesorption isotherms are commonly used to measure the textural properties of SBA15. The isotherm of the samples (Figure 5A) showed typical irreversible type IV adsorption features with an $\mathrm{H} 1$ hysteresis loop, which is a characteristic of mesoporous material, as defined by the International Union of Pure and Applied Chemistry, ${ }^{32}$ and indicative of highly ordered structures with a narrow mesoporous size distribution. ${ }^{33}$ Figure 5B shows a representative highresolution TEM image of SBA15 confirming an ordered
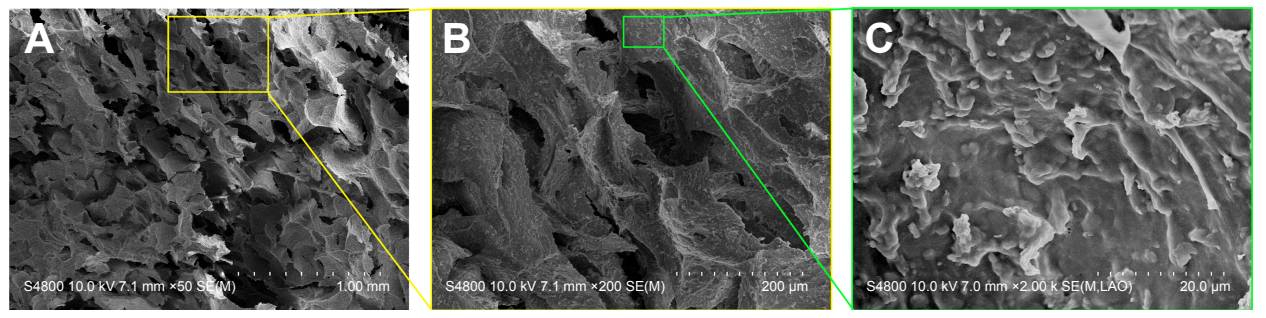

Figure 3 Morphology of SF/SBAI5 scaffold imaged by FE-SEM. (A) SF/SBAI5 scaffold; (B) and (C) respectively show the zoomed in morphology of the pointed area (yellow and green).

Abbreviations: FE-SEM, field emission scanning electron microscope; SF, silk fibroin. 

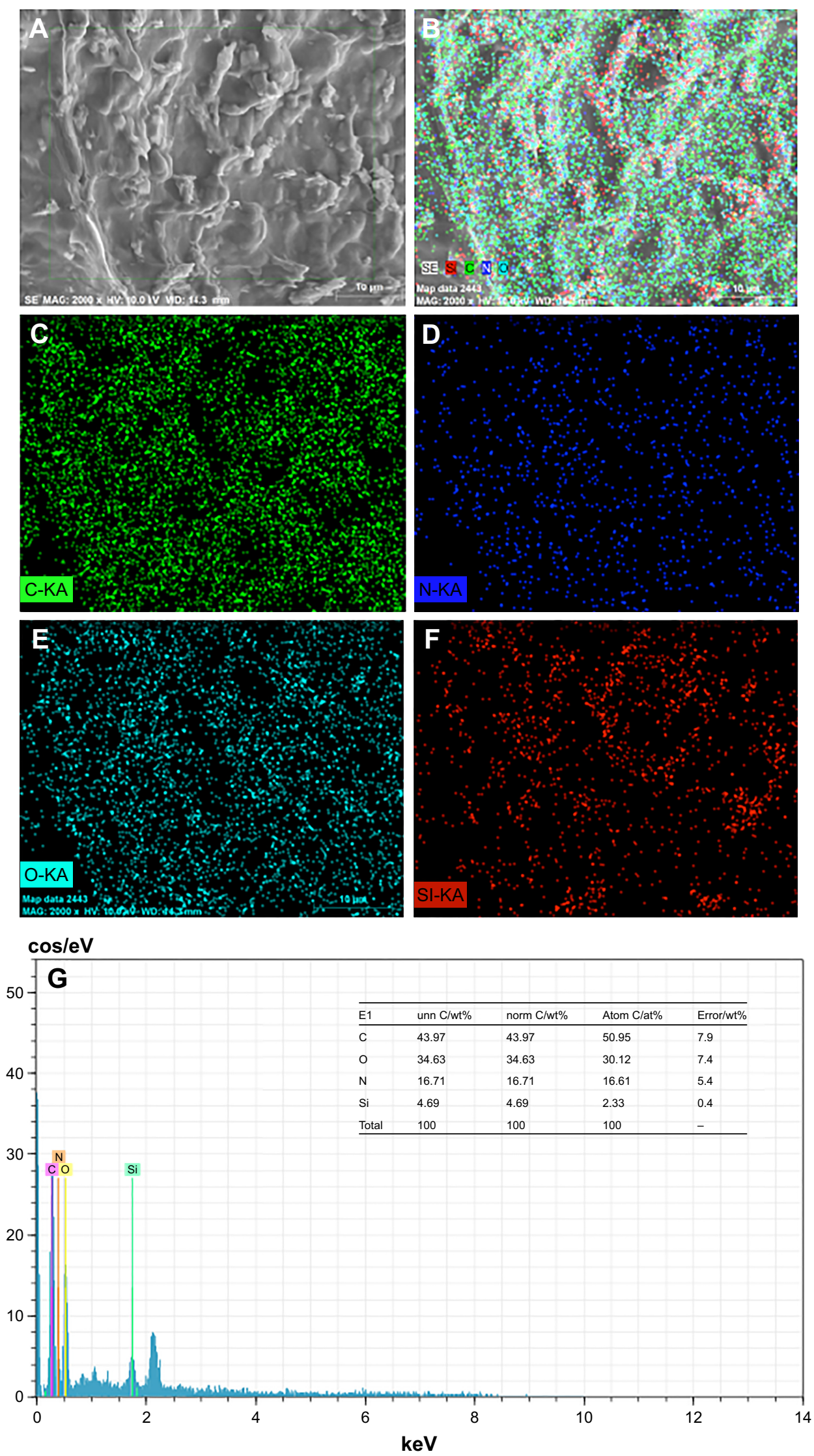

Figure 4 (A) Selected area on SF/SBAI5 scaffold and the corresponding (B) elemental distribution mapped by EDS-XRS. Elemental distributions of (C) C, (D) N, and (E) $O$ indicate the presence of SF. Elemental distribution of (F) Si indicates the presence of SBAI5. (G) Quantitative analysis of all the elements on the selected scaffold surface. Abbreviations: at\%, atomic percent; C, carbon; EDS-XRS, energy-dispersive X-ray spectrometry; N, nitrogen; norm, normalization; O, oxygen; SF, silk fibroin; Si, silicon; unn, unnormalization. 
A

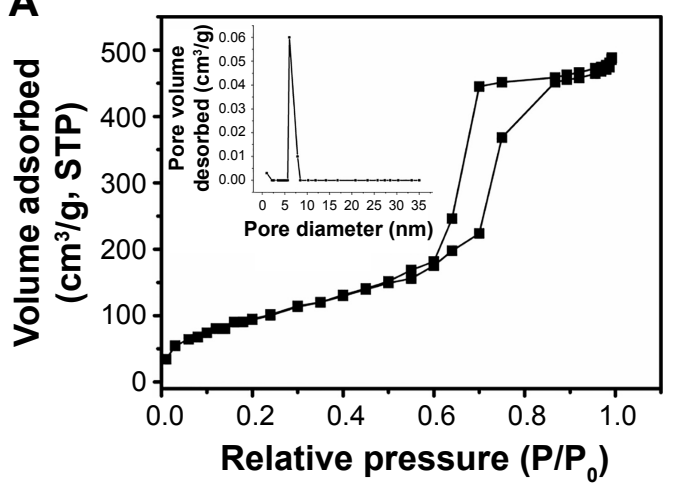

B

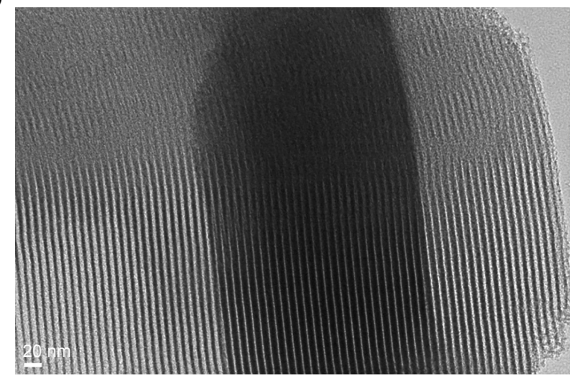

Figure 5 (A) Nitrogen adsorption-desorption isotherms, pore size distribution plots (inset), and (B) TEM images of the prepared SBA 5 mesoporous silica. Abbreviations: STP, standard temperature and pressure; TEM, transmission electron microscopy.

array structure with a relatively uniform pore size. As shown in Figure 5B, the TEM image of calcined SBA15 clearly revealed parallel well-ordered tubular mesoporous and straight lattice fringes. Calculating the BET surface areas and the mesoporous parameters of SBA15 according to $\mathrm{BJH}$ analysis revealed that the average mesoporous size was $6.8 \mathrm{~nm}$, and the BET surface area and pore volume were $823 \mathrm{~m}^{2} / \mathrm{g}$ and $1.22 \mathrm{~m}^{3} / \mathrm{g}$, respectively, which were in accordance with previous reports. ${ }^{34}$

A

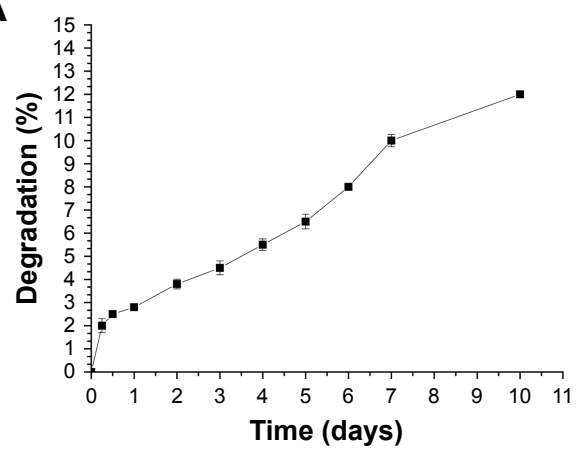

C

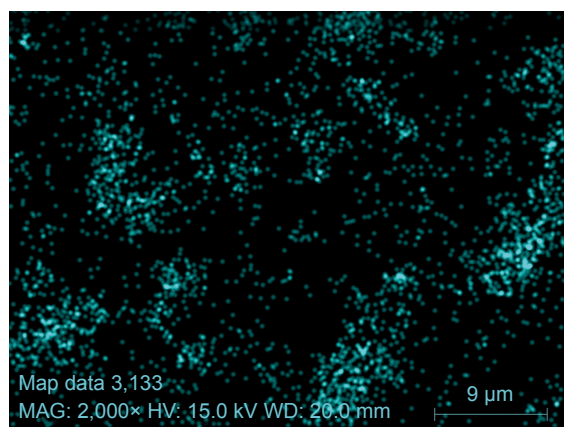

\section{In vitro degradation study}

Figure 6 shows an in vitro degradation study of the composite scaffold SF/SBA15. The degradation profile showed $\sim 10 \%$ dry weight loss in 10 days (Figure 6A). To evaluate the influence of degradation on microstructure of the composite scaffold, the samples at Day 10 were analyzed by FE-SEM, EDS-XRS, BET, and TEM. Cross-section morphology of the composite scaffold SF/SBA15 imaged by FE-SEM shows the similar porous structures after 10 days of immersion in PBS

\section{B}

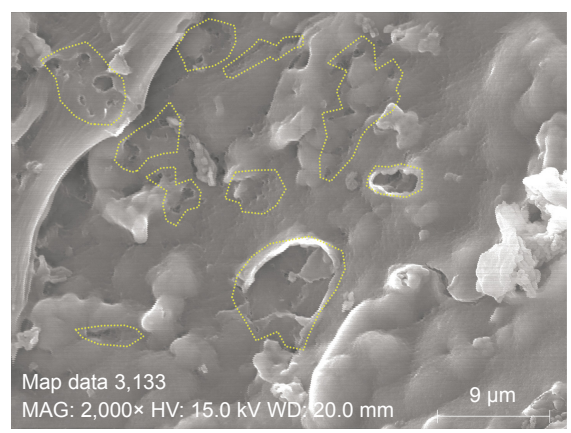

D

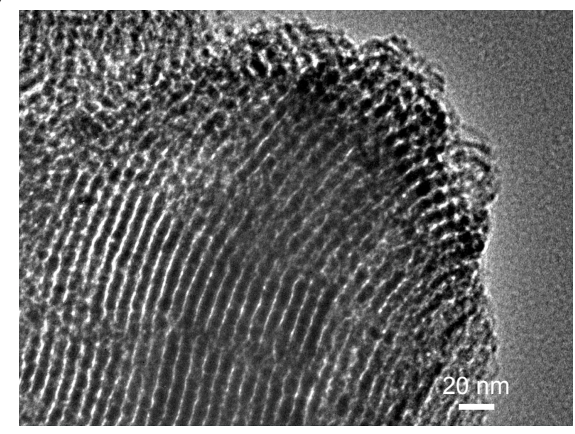

Figure 6 (A) The degradation profile of the SF/SBAI5 scaffold over 10 days. (B) Cross-section morphology of the composite scaffold imaged by FE-SEM after 10 days of in vitro degradation study, on which the yellow dotted circles are the newly presented small pores provided by the degradation of the composite scaffold. Silicon elemental distribution (C) on the scaffold surface is mapped by EDS-XRS, and the microstructure of SBAI5 (D) in the composite scaffold after 10 days of degradation is shown by TEM image.

Abbreviations: EDS-XRS, energy-dispersive X-ray spectrometry; FE-SEM, field emission scanning electron microscope; SF, silk fibroin; TEM, transmission electron microscopy. 
as the untreated SF/SBA15 scaffold (Figure 6B). However, compared with the integrated wall of the untreated SF scaffold (Figure 3C), many newly presented small pores with a diameter of $0.2-8 \mu \mathrm{m}$ are observed on the wall of the SF scaffold after 10 days of degradation study (Figure 6B). Silicon elemental distribution on the scaffold surface of SF/SBA15 after 10 days of degradation study determined by EDS-XRS mapping (Figure 6C) is identical with the untreated scaffold (Figure 4F), which indicates that the content and location of SBA15 on the wall of the SF scaffold are maintained. TEM image of the pulverized sample after 10 days of degradation study (Figure 6D) shows that there still involve the tubular mesopores with clear edges in an ordered array. It indicates that the SF scaffold plays an important role in protecting SBA15 from degradation when compared with the pure SBA15, which may degrade in vitro in 24 hours. ${ }^{35}$ According to the result of nitrogen adsorption-desorption isotherms, after 10 days of degradation study, the average mesoporous size of SBA15 in the composite scaffold was $5.1 \mathrm{~nm}$, and the BET surface area was $113 \mathrm{~m}^{2} / \mathrm{g}$. Since SBA15 were embedded in the SF scaffold and SF may block some pores of SBA15, these data values were slightly lower than those of the pure SBA15 (mesoporous size $=6.8 \mathrm{~nm}$, BET surface area $=823 \mathrm{~m}^{2} / \mathrm{g}$ ).

\section{BMP2 and IC in vitro release profiles}

The release kinetics of BMP2 were observed for different types of composite scaffolds (Figure 7A). All groups showed an initial burst release of BMP2 during the first 24 hours to $\sim 60 \%$ of the cumulative release with a concentration of $\sim 1 \mu \mathrm{g} / \mathrm{mL}$, which is mainly attributed to the physically adsorbed protein on the surface of microstructured pores in SF scaffolds. The microporous structure of the SF scaffolds was large enough to ensure that BMP2 easily passes through. ${ }^{11}$ Over the following 6 days, sustained gradual release of the protein was observed. Although BMP2 easily loses its bioactivity due to its short half-life, ${ }^{3}$ its retention was prolonged when it was loaded into the composite scaffold, as scaffold effectively retained the protein. In general, all curves in Figure $7 \mathrm{~A}$ are consistent, indicating that the existence of IC and SBA15 had little influence on the release rate of BMP2, which suggests that the BMP2 was located in the micropores of the SF scaffold, where SBA15 can exert little influence (Figure 3).

In addition, the release kinetics of IC were determined for different types of composite scaffolds (Figure 7B). All scaffolds exhibited an initial burst release during the first 24 hours. The $\mathrm{SF}_{\mathrm{BMP} 2 / \mathrm{IC}} / \mathrm{SBA} 15$ scaffold with IC located in the microporous structure of the SF component showed $95.1 \% \pm 1.0 \%$ burst release that reached the concentration of $34.3 \pm 0.4 \mu \mathrm{M}$ compared with $71.8 \% \pm 1.7 \%$ burst release that reached $25.9 \pm 0.6 \mu \mathrm{M}$ from the SBA15 ${ }_{\text {IC }}$ scaffold containing IC in its own mesoporous channels. The SF/SBA15 IC and $\mathrm{SF}_{\mathrm{BMP} 2} / \mathrm{SBA} 15_{\text {IC }}$ scaffolds both achieved $\sim 64.2 \%$ burst release that reached the concentration of $23 \mu \mathrm{M}$; in these systems, IC was first incorporated into the mesoporous channels of SBA15 and then embedded into the micropores of SF. These findings suggest that the IC release from the composite scaffold can be fine-tuned by varying the system structure. The $\mathrm{SF}_{\mathrm{BMP} / \mathrm{IC}} / \mathrm{SBA} 15$ scaffolds exhibited nearly no controlled release of IC, as almost all of the IC was loaded in the micropores of the SF component, whose pore size
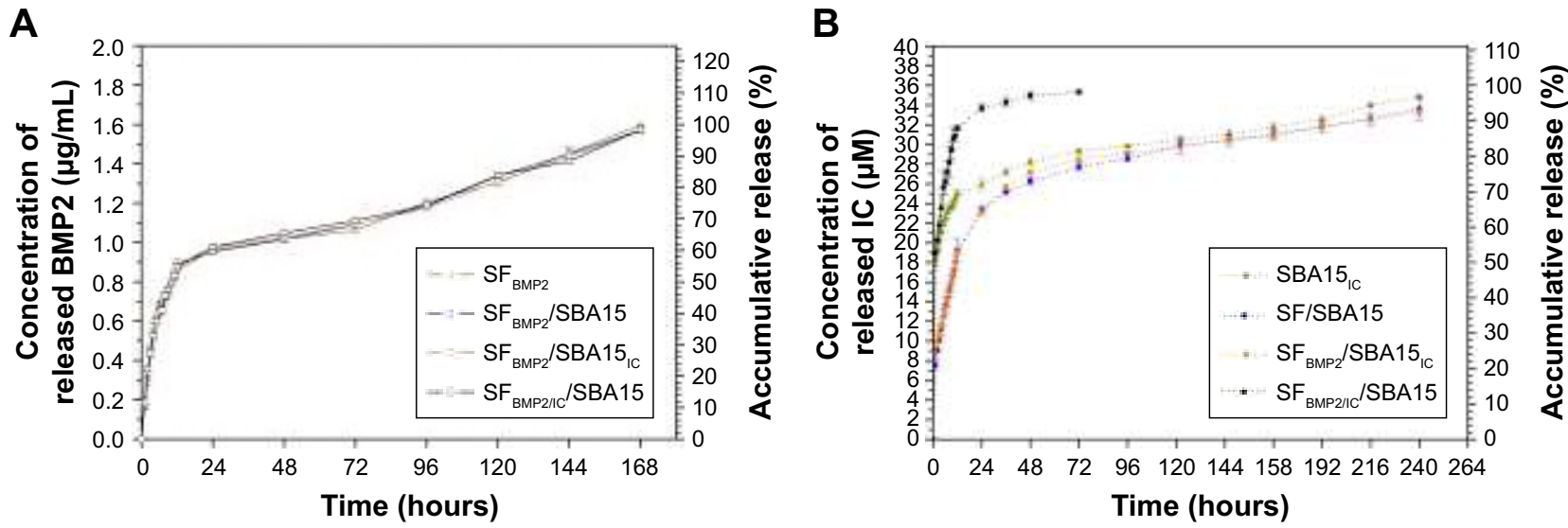

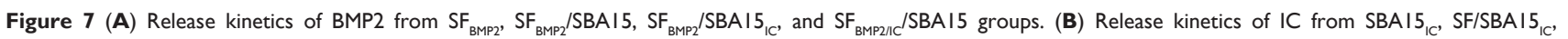
$\mathrm{SF}_{\mathrm{BMP2}} / \mathrm{SBA} / 5_{\mathrm{IC}}$, and $\mathrm{SF}_{\mathrm{BMP} / / \mathrm{C}} / \mathrm{SBA} 15$ groups.

Note: The scale plate on the left side is the concentration of released BMP2 $(\mu \mathrm{g} / \mathrm{mL})$ or icariin $(\mu \mathrm{M})$, and on the right side is the cumulative release which expressed as the percentage of total loaded protein or drug. Abbreviations: IC, icariin; SF, silk fibroin. 
was too large to maintain a local reserve of $\mathrm{IC}$, which is a small-molecule drug. When IC was embedded into SBA15 nanoparticles, the initial burst of IC decreased, and a longer sustained release was observed, which was possibly related to the mesoporous structure of SBA15 and an interaction between the drug and the mesoporous silica by hydrogen bonding. ${ }^{36}$ The SF/SBA15 IC and $\mathrm{SF}_{\text {BMP2 }} / \mathrm{SBA} 15_{\text {IC }}$ scaffolds exhibited the lowest rates of initial burst release of IC among all groups; this burst was followed by a sustained release. This result is explained by the fact that, when IC spreads from the composite scaffold, it must pass through not only the mesoporous channels of SBA15, but also the interconnected microporous structure of the SF scaffold. The similar release trends of these two groups (SF/SBA15 $5_{\mathrm{IC}}$ and $\mathrm{SF}_{\mathrm{BMP} 2} / \mathrm{SBA} 15_{\mathrm{IC}}$ ) also showed that the existence of BMP2 had little influence on the release rate of IC. A gradual release after the burst release of IC was detected in nearly every group. Subsequently, sustained release to $96.6 \% \pm 0.8 \%$ that reached the concentration of $34.8 \pm 0.3 \mu \mathrm{M}$ occurred from the SBA15 scaffolds over 10 days compared with $92.4 \% \pm 0.8 \%$ reached the concentration of $33.3 \pm 0.3 \mu \mathrm{M}$ from the $\mathrm{SF} / \mathrm{SBA} 15_{\mathrm{IC}}$ and $\mathrm{SF}_{\mathrm{BMP} 2} / \mathrm{SBA} 15_{\mathrm{IC}}$ scaffolds over 10 days; however, the $\mathrm{SF}_{\mathrm{BMP} 2 / \mathrm{C}} / \mathrm{SBA} 15$ composite scaffold released the remaining drug completely in 3 days. This finding was attributed to the construction of the composite SF/SBA15 scaffold. Due to its highly organized porosity, large surface area, large pore volume, controlled pore size, and wall thickness, the ordered mesoporous SBA15 is considered a prominent candidate for drug delivery. ${ }^{21,37}$ Furthermore, when SBA15 is embedded into microstructured SF scaffolds, the structure presents a highly porous, interconnected morphology that prolongs the local retention of BMP2 and IC.

BMP2 may easily lose its bioactivity due to its short half-life, ${ }^{3}$ and IC is absorbed in a short time due to its small molecular size. ${ }^{21}$ However, when these components were incorporated into the hierarchical porous delivery system, the release time was extended to 7 and 11 days, showing that the micro/meso hierarchical porous delivery system composed of micropores of an SF scaffold and mesopores of SBA15 can prolong the retention of BMP2 and IC, which is a critical factor in the construction of bone tissue engineering systems. ${ }^{38}$

\section{Cell biocompatibility}

Figure 8 shows the cell biocompatibility of the composite scaffold SF/SBA15. The image of live/dead cells, which indicates the cell viability, showed that almost all of the BMSCs on the scaffolds maintained their activities (Figure 8A).
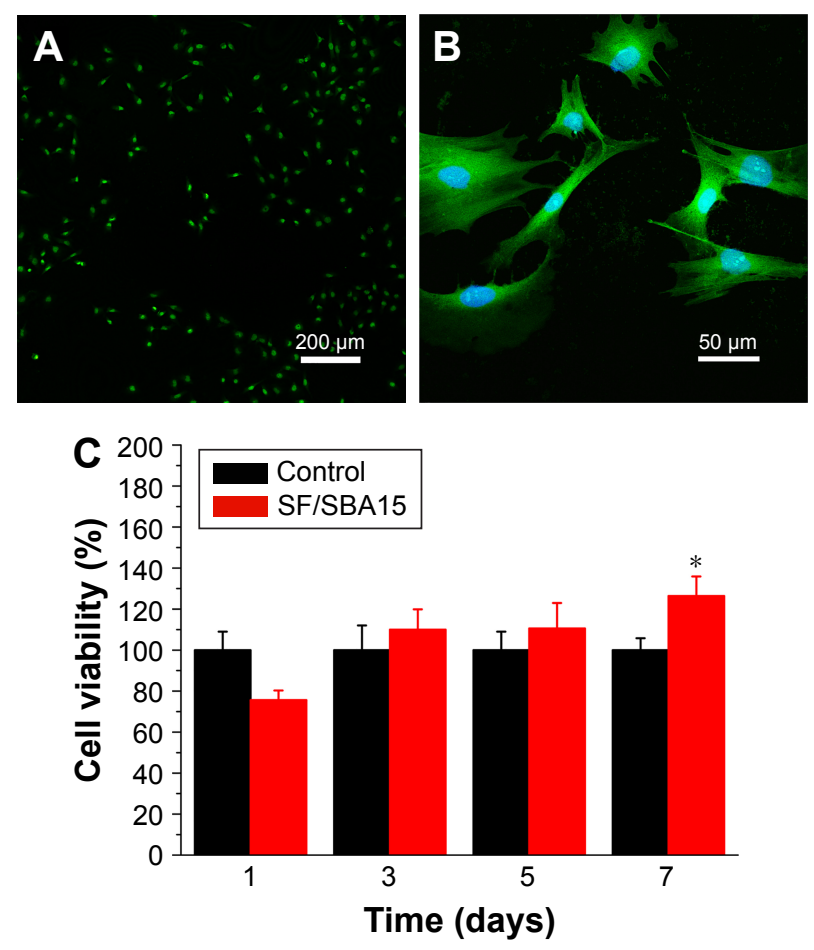

Figure 8 Cell biocompatibility of the composite scaffold SF/SBAI5. (A) Live/dead images of BMSCs cultured with the scaffold after 24 hours. (B) Cell morphology on the scaffold after 24 hours. (C) Cell viability in the scaffold detected by MTS analysis at Days I, 3, 5, and 7. ${ }^{*} p<0.05$ is the significant difference to the corresponding positive control.

Abbreviations: BMSC, bone marrow mesenchymal stem cell; SF, silk fibroin.

The cell adhesion and ingrowth of BMSCs were studied 24 hours after seeding on the surface of the composite scaffolds (Figure 8B). The demonstrated features were consistent with adhesion to the scaffold with elongated shapes and a few thin pseudopodia. The elongated morphology showed the good attachment and spreading of BMSCs. At Day 7, compared with the cell viability of the control group, the cell viability (MTS) in the scaffolds was significantly increased (Figure 8C). All of the biocompatibility results showed that the scaffolds are nontoxic and promote cell proliferation and attachment.

\section{In vitro mineralization}

In vitro mineralization was measured at 7 days, 14 days, and 28 days and showed that strongly stained and correlative mineral features were formed in different groups. ALP staining and quantitation (Figure 9) and Alizarin red staining (Figure 10) indicated that the $\mathrm{SF}_{\mathrm{BMP} 2} / \mathrm{SBA} 15_{\mathrm{IC}}$ scaffold promoted better osteogenic differentiation than the other groups, likely because the silicon-containing SBA15 loaded into the SF scaffold is osteoinductive for bone formation. ${ }^{39}$ Meanwhile, the $\mathrm{SF}_{\mathrm{BMP} 2} / \mathrm{SBA} 15_{\mathrm{IC}}$ scaffolds exhibited the most strongest osteogenesis effect with the matched release rates 

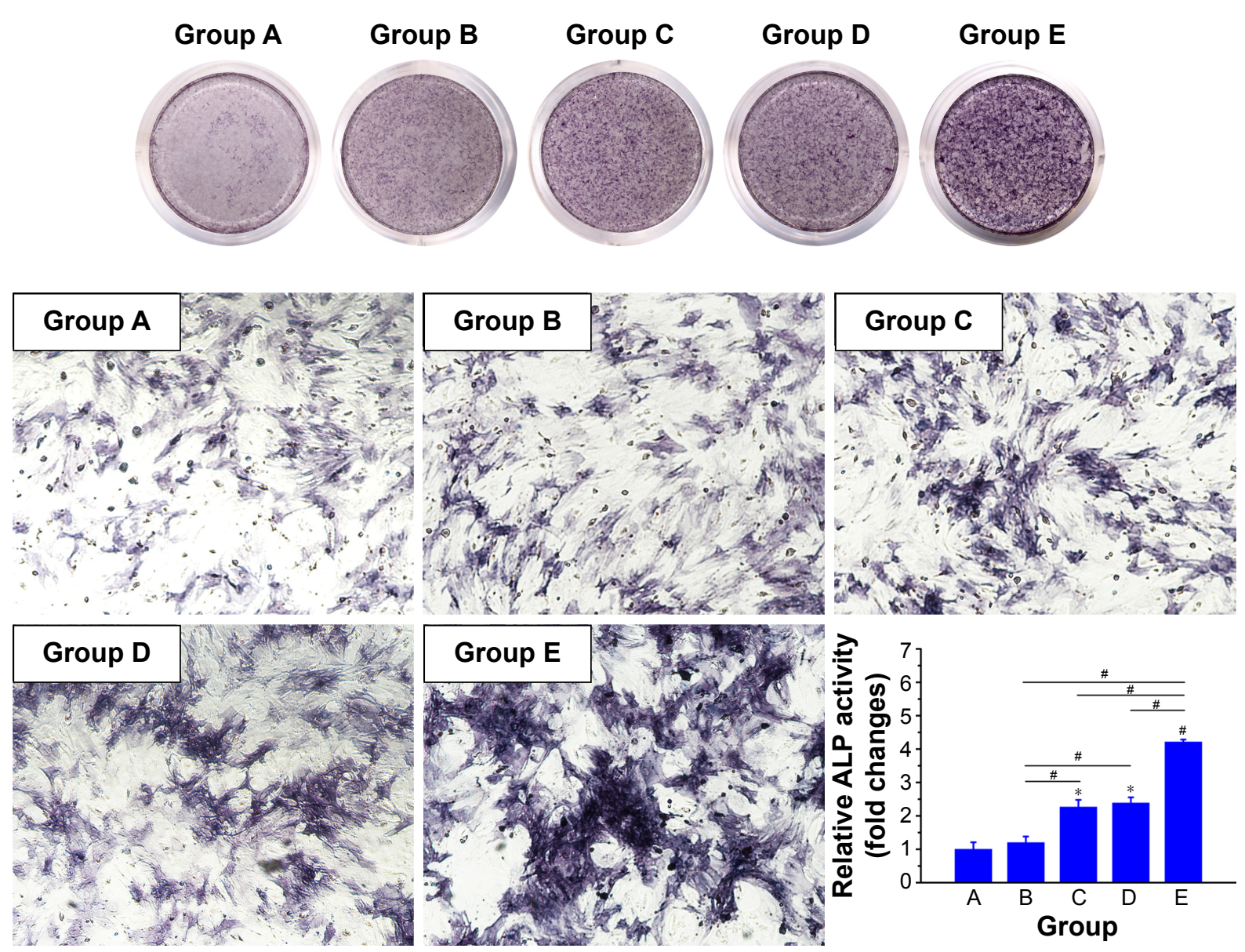

Figure 9 ALP staining and the quantitation analysis. Rat BMSCs were cocultured with a transwell system loaded with different scaffolds for 7 d. Group A: SF/SBAI5, Group B: SF/

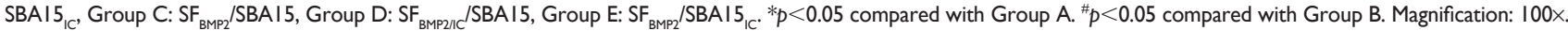
Abbreviations: ALP, alkaline phosphatase; BMSC, bone marrow mesenchymal stem cell; IC, icariin; SF, silk fibroin.

of BMP2 and IC. ALP quantitation analysis showed the significant increase in the $\mathrm{SF}_{\mathrm{BMP} 2} / \mathrm{SBA} 15_{\text {IC }}$ group at Day 7 , most likely due to the contributing synergistic effects of IC and BMP2.

\section{Quantitative real-time PCR}

Quantitative real-time PCR analysis of BMSCs cocultured with different payload composite scaffolds was performed to evaluate the expression levels of osteogenic differentiation markers (Figure 11). RUNX2, which is a known osteoblast transcriptional activator and a key regulator of osteoblast differentiation, plays an important role in regulating the expression of major osteoblast genes and in maintaining the functions of differentiated osteoblasts at an early stage. ${ }^{20}$ $A L P$ is a marker of early osteogenic differentiation and commits BMSCs toward the osteoblastic phenotype..$^{40} \mathrm{Col} \mathrm{I}$ is reported to be a typical marker associated with the formation of the extracellular matrix at the initial stage of osteogenic differentiation. ${ }^{40} O C N$, the most specific and late marker of osteogenic differentiation, is associated with matrix deposition and mineralization. ${ }^{20}$ Quantitative real-time PCR analysis showed that, compared with the control group (TCPs), in the SF/SBA15 group, the expression levels of $R U N X 2, A L P$, Col I, and $O C N$ were slightly upregulated, which was attributed to the outstanding biocompatibility of the SF scaffold ${ }^{25}$ and the potential pro-osteogenesis function of SBA15. ${ }^{39}$ Compared with the control group and the SF/SBA15 scaffolds, the composite scaffolds loaded with BMP2, IC, or both all exhibited higher osteogenic gene expression levels in the cocultured BMSCs, whereas different assemblies with varying locations of BMP2 or IC in the scaffolds promoted different degrees of enhancement. All the relative gene expression levels of the selected bone markers were promoted in the single payload groups, composite $\mathrm{SF}_{\mathrm{BMP} 2} / \mathrm{SBA} 15$ scaffold, and SF/SBA15 ${ }_{\mathrm{IC}}$ scaffold. All of the analyzed markers increased significantly between the control group and the single payload groups. Furthermore, compared with the values in the SF/SBA15 group, the expression levels of all the analyzed markers were significantly upregulated in the $\mathrm{SF}_{\mathrm{BMP} 2} / \mathrm{SBA} 15$ group, and 


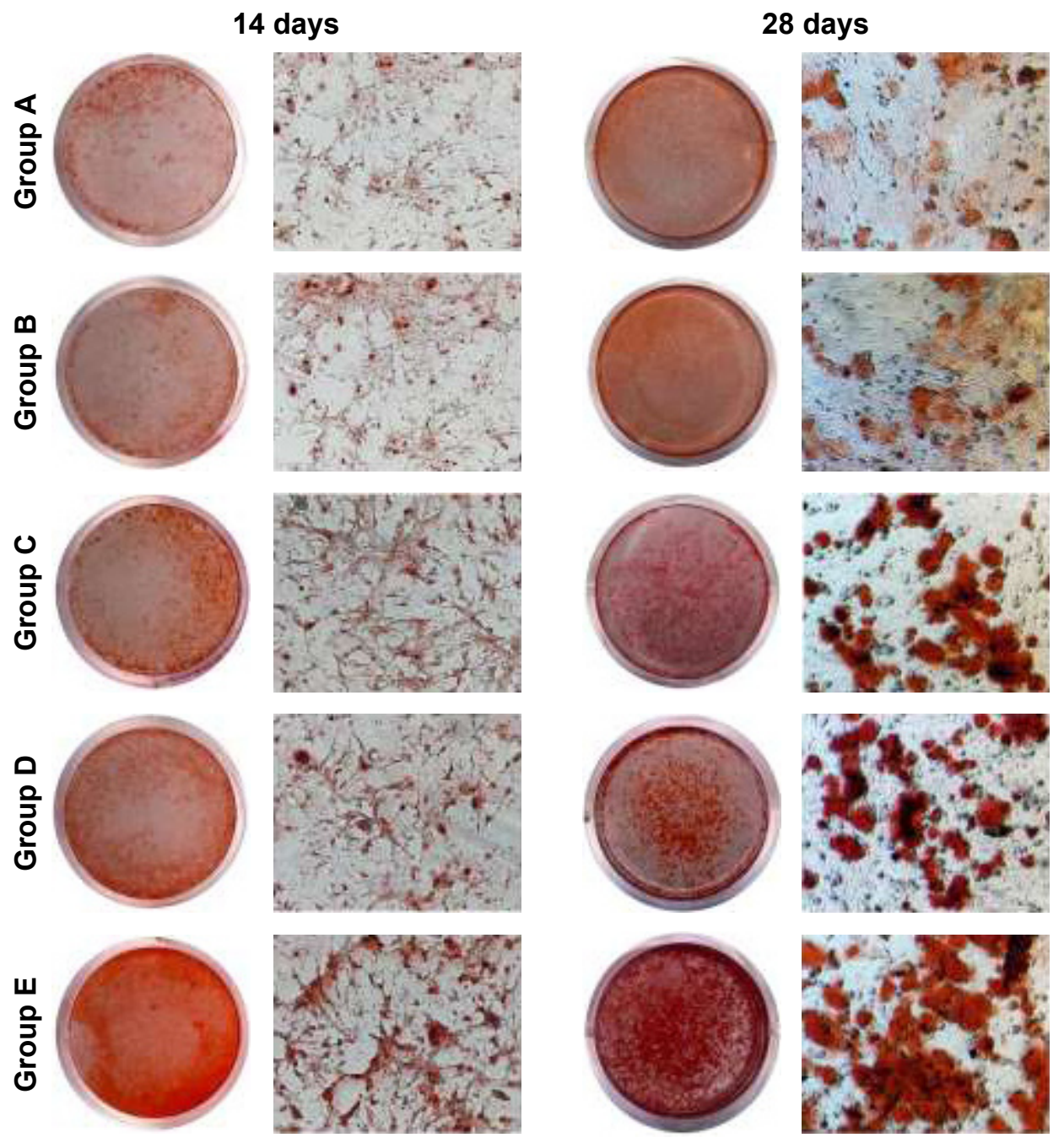

Figure 10 Alizarin red staining. Rat BMSCs were cocultured with transwell loaded with different scaffolds for 14 and 28 days. Group A: SF/SBAI5, Group B: SF/SBAI5 Group C: $\mathrm{SF}_{\mathrm{BMP2}} / \mathrm{SBA} 15$, Group D: $\mathrm{SF}_{\mathrm{BMP}_{2} / \mathrm{C}} / \mathrm{SBA} 15$, Group E: $\mathrm{SF}_{\mathrm{BMP}_{2}} / \mathrm{SBAI} 5_{\mathrm{IC}}$. Magnification: $100 \times$.

Abbreviations: $B M S C$, bone marrow mesenchymal stem cell; $I C$, icariin; SF, silk fibroin.

the expression levels of $\mathrm{Col} I$ and $O C N$ were upregulated at 7 days in the SF/SBA15 IC group. However, compared with the $\mathrm{SF} / \mathrm{SBA} 15_{\mathrm{IC}}$ group, $\mathrm{SF}_{\mathrm{BMP}} / \mathrm{SBA} 15$ displayed significantly higher expression levels for all four osteogenic genes at 7 days, which was equivalent to the single payload group and confirms that BMP2 facilitates osteogenic differentiation and functions as the predominate effective growth factor. As the well-loaded and controlled release group (Figure 7), the $\mathrm{SF}_{\mathrm{BMP} 2} / \mathrm{SBA} 15_{\mathrm{IC}}$ composite scaffold had different locations for BMP2 and IC in the microporous structure of the SF scaffold and in the mesoporous channels of SBA15, respectively. In this group, the expression of all the selected osteogenic genes showed significantly higher increases than those in the control group and the SF/SBA15 group, which revealed that the $\mathrm{SF}_{\mathrm{BMP}^{2}} / \mathrm{SBA} 15_{\mathrm{IC}}$ scaffold could effectively maintain the bioactivity of BMP2 and IC and control their release.
Compared with the single payload composite scaffold $\left(\mathrm{SF}_{\mathrm{BMP} 2} / \mathrm{SBA} 15\right.$ and $\left.\mathrm{SF} / \mathrm{SBA} 15_{\mathrm{IC}}\right)$, the $\mathrm{SF}_{\mathrm{BMP}_{2}} / \mathrm{SBA} 15_{\mathrm{IC}}$ scaffold also showed notably enhanced expression of all four genes at 3 days and of $R U N X 2$ and $A L P$ at 7 days. Therefore, simultaneously controlling the release of BMP2 and IC more effectively influenced osteogenesis than did the controlled release of a single cue. In the composite $\mathrm{SF}_{\mathrm{BMP} 2 / \mathrm{C}} / \mathrm{SBA} 15$ scaffold with IC and BMP2 simultaneously embedded into the microporous structure of the SF scaffold, the expression of the four selected genes was higher than that in the control group, the SF/SBA15 group, and the SF/SBA15 IC group at 3 and 7 days, whereas, compared with the control group, the $\mathrm{SF}_{\text {BMP2 }} / \mathrm{SBA} 15$ group was not significantly different. This result implied that although the addition of IC to the $\mathrm{SF}_{\text {BMP2/IC }}$ SBA15 scaffold slightly promoted osteogenesis, the final augmentation of gene expression primarily originated from 

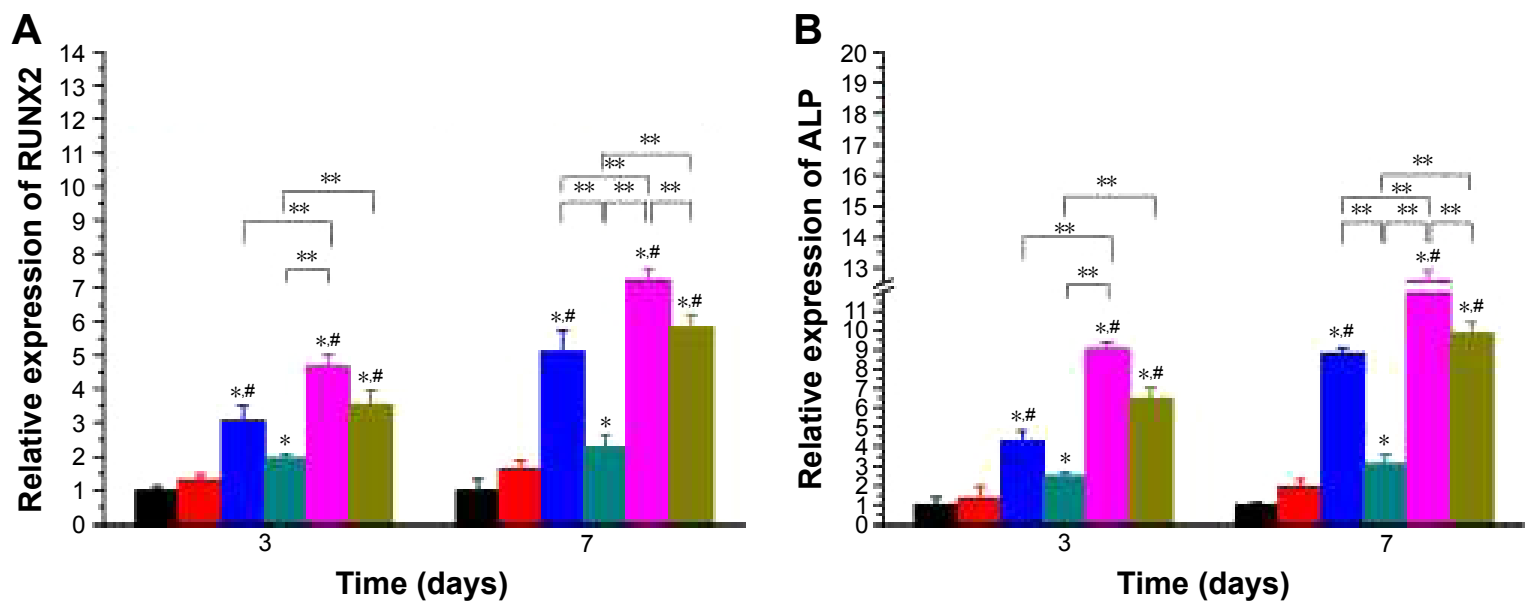

Time (days)
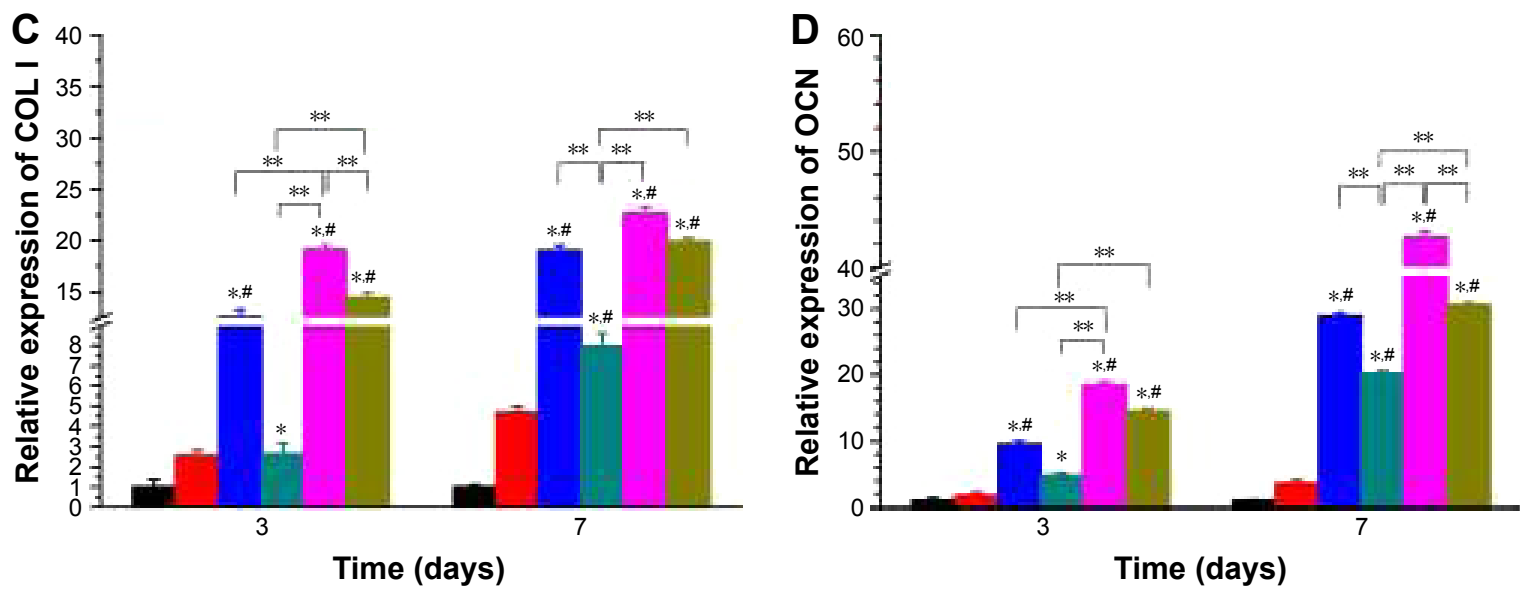

\begin{tabular}{|lll|}
\hline Control & $\mathrm{SF} / \mathrm{SBA} 15$ & $\mathrm{SF}_{\mathrm{BMP2}_{2}} / \mathrm{SBA} 15$ \\
$\mathrm{SF} / \mathrm{SBA} 15_{\mathrm{IC}}$ & $\mathrm{SF}_{\mathrm{BMP2}} / \mathrm{SBA} 15_{\mathrm{IC}}$ & $\mathrm{SF}_{\mathrm{BMP} 2 / \mathrm{C}} / \mathrm{SBA} 15$ \\
\hline
\end{tabular}

Figure II Relative gene expression levels of osteogenic differentiation markers (A) RUNX2, (B) ALP, (C) COL I, and (D) OCN in rat BMSCs cocultured with different scaffolds at Days 3 and $7 .{ }^{*} p<0.05$, compared with the control group; ${ }^{* *} p<0.05$, compared with the selected groups; ${ }^{*} p<0.05$, compared with SF/SBAI5 group. Abbreviations: ALP, alkaline phosphatase; BMSC, bone marrow mesenchymal stem cell; IC, icariin; SF, silk fibroin.

the function of BMP2. Moreover, compared with the values in the $\mathrm{SF}_{\mathrm{BMP} 2} / \mathrm{SBA} 15_{\text {IC }}$ group, the activity of the osteogenic marker Col I was significantly reduced at 3 days, and the activities of $R U N X 2, A L P$, and $O C N$ were reduced at 7 days in the $\mathrm{SF}_{\mathrm{BMP} 2 / \mathrm{IC}} / \mathrm{SBA} 15$ composite scaffold. These results showed that matched controlled release of BMP2 and IC loaded in different structures of the composite scaffold not only maintained osteogenesis, but also exerted positive effects on bone formation. As the micropores of the SF scaffold accelerated the release rate of IC, an obvious initial burst release from the $\mathrm{SF}_{\mathrm{BMP} 2 / \mathrm{C}} / \mathrm{SBA} 15$ scaffold was revealed by the release profile (Figure 7B). Hence, the limited interaction time between IC and BMP2 decreased their synergistic effect on osteogenesis, which confirmed that these compounds can coordinate osteogenesis only in conditions with a matched release rate, and the delivery of multiple biomolecules can induce synergistic effects on enhancing bone tissue regeneration. ${ }^{1,41}$

\section{Conclusion}

In the present study, a micro/meso hierarchical porous delivery system was developed by combining SBA15 nanoparticles with microstructured SF scaffolds, which could well control the dual delivery of BMP2 and IC and synergistically promote their bioactivities. The results demonstrated that mesoporous SBA15 could optimize the efficacy of SF scaffolds by significantly prolonging local retention of the protein and drug. The developed composite scaffold presented an appropriate physical structure, good bioactivity and biocompatibility, and a proper degradation rate. Moreover, it activated osteogenic differentiation in vitro and, therefore, has the potential to promote bone regeneration in cases of bone defects. 


\section{Acknowledgments}

This project was supported by the National Natural Science Foundation of China (Numbers 31500787, 81600828, 81620108006, and 81430012), the National Key Research and Development Program of China (2016YFC1102900), Science and Technology Commission of Shanghai Municipality (15410722700) and Medicine and Engineering Cross Research Foundation of Shanghai Jiao Tong University (YG2015ZD06), and Program for Innovation Team Building at Institutions of Higher Education in Chongqing in 2016 (CXTDG201602006).

\section{Disclosure}

The authors report no conflicts of interest in this work.

\section{References}

1. Yu X, Khalil A, Dang PN, Alsberg E, Murphy WL. Multilayered inorganic microparticles for tunable dual growth factor delivery. $A d v$ Funct Mater. 2014;24(20):3082-3093.

2. Hokugo A, Saito T, Li A, Sato K, Tabata Y, Jarrahy R. Stimulation of bone regeneration following the controlled release of water-insoluble oxysterol from biodegradable hydrogel. Biomaterials. 2014;35(21): $5565-5571$.

3. Li L, Zhou G, Wang Y, Yang G, Ding S, Zhou S. Controlled dual delivery of BMP-2 and dexamethasone by nanoparticle-embedded electrospun nanofibers for the efficient repair of critical-sized rat calvarial defect. Biomaterials. 2015;37:218-229.

4. Agrawal V, Sinha M. A review on carrier systems for bone morphogenetic protein-2. J Biomed Mater Res B Appl Biomater. 2017;105(4): 904-925.

5. Gautschi OP, Frey SP, Zellweger R. Bone morphogenetic proteins in clinical applications. ANZ J Surg. 2007;77(8):626-631.

6. Kaneko H, Arakawa T, Mano H, et al. Direct stimulation of osteoclastic bone resorption by bone morphogenetic protein (BMP)-2 and expression of BMP receptors in mature osteoclasts. Bone. 2000;27(4):479-486.

7. Li B, Yoshii T, Hafeman AE, Nyman JS, Wenke JC, Guelcher SA. The effects of rhBMP-2 released from biodegradable polyurethane/ microsphere composite scaffolds on new bone formation in rat femora. Biomaterials. 2009;30(35):6768-6779.

8. Langenfeld EM, Kong Y, Langenfeld J. Bone morphogenetic protein 2 stimulation of tumor growth involves the activation of Smad-1/5. Oncogene. 2006;25(5):685-692.

9. Thanyaphoo S, Kaewsrichan J. A new biocompatible delivery scaffold containing heparin and bone morphogenetic protein 2. Acta Pharm. 2016;66(3):373-385.

10. Cao L, Wang J, Hou J, Xing W, Liu C. Vascularization and bone regeneration in a critical sized defect using 2-N,6-O-sulfated chitosan nanoparticles incorporating BMP-2. Biomaterials. 2014;35(2):684-698.

11. Zhang W, Wang X, Wang S, et al. The use of injectable sonicationinduced silk hydrogel for VEGF165 and BMP-2 delivery for elevation of the maxillary sinus floor. Biomaterials. 2011;32(35):9415-9424.

12. Wei H, Zili L, Yuanlu C, et al. Effect of icariin on bone formation during distraction osteogenesis in the rabbit mandible. Int J Oral Maxillofac Sury. 2011;40(4):413-418.

13. Zhang G, Qin L, Hung WY, et al. Flavonoids derived from herbal Epimedium Brevicornum Maxim prevent OVX-induced osteoporosis in rats independent of its enhancement in intestinal calcium absorption. Bone. 2006;38(6):818-825.

14. Song L, Zhao J, Zhang X, Li H, Zhou Y. Icariin induces osteoblast proliferation, differentiation and mineralization through estrogen receptor-mediated ERK and JNK signal activation. Eur J Pharmacol. 2013;714(1-3):15-22.
15. Wei Q, He M, Chen M, et al. Icariin stimulates osteogenic differentiation of rat bone marrow stromal stem cells by increasing TAZ expression. Biomed Pharmacother. 2017;91:581-589.

16. Wu Y, Xia L, Zhou Y, et al. Evaluation of osteogenesis and angiogenesis of icariin loaded on micro/nano hybrid structured hydroxyapatite granules as a local drug delivery system for femoral defect repair. J Mater Chem B. 2015;3:4871-4883.

17. Wu Y, Xia L, Zhou Y, Xu Y, Jiang X. Icariin induces osteogenic differentiation of bone mesenchymal stem cells in a MAPK-dependent manner. Cell Prolif. 2015;48(3):375-384.

18. Kasoju N, Bora U. Silk fibroin in tissue engineering. Adv Healthc Mater. 2012;1(4):393-412.

19. Andreza S, Martins E. Ordered mesoporous silica carrier system applied in nanobiothecnology. Braz Arch Biol Technol. 2005;48(2):243-250.

20. Dai C, Guo H, Lu J, Shi J, Wei J, Liu C. Osteogenic evaluation of calcium/magnesium-doped mesoporous silica scaffold with incorporation of rhBMP-2 by synchrotron radiation-based $\mu \mathrm{CT}$. Biomaterials. 2011;32(33):8506-8517.

21. Wang Y, Zhao Q, Han N, et al. Mesoporous silica nanoparticles in drug delivery and biomedical applications. Nanomedicine. 2015;11(2): 313-327.

22. Shi S, Jiang W, Zhao $\mathrm{T}$, et al. The application of nanomaterials in controlled drug delivery for bone regeneration. J Biomed Mater Res. 2015; 103(12):3978-3992.

23. Cheng L, He X, Che Y, Che H, Qian M. Osteogenesis-promoting activity of composites SBA-15 mesoporous particles carrying oxytocin in vitro and in vivo. J Biol Regul Homeost Agents. 2017;31(1):157-162.

24. Gallo JM, Bisio C, Gatti G, Marchese L, Pastore HO. Physicochemical characterization and surface acid properties of mesoporous [Al]-SBA-15 obtained by direct synthesis. Langmuir. 2010;26(8):5791-5800.

25. Fini M, Motta A, Torricelli $P$, et al. The healing of confined critical size cancellous defects in the presence of silk fibroin hydrogel. Biomaterials. 2005;26(17):3527-3536.

26. Zhao D, Feng J, Huo Q, et al. Triblock copolymer syntheses of mesoporous silica with periodic 50 to 300 angstrom pores. Science. 1998; 279(5350):548-552.

27. Mellaerts R, Aerts CA, Humbeeck J, Augustijns P, Van den Mooter G, Martens JA. Enhanced release of itraconazole from ordered mesoporous SBA-15 silica materials. Chem Commun (Camb). 2007;(13): 1375-1377.

28. Yucel T, Lovett ML, Kaplan DL. Silk-based biomaterials for sustained drug delivery. J Control Release. 2014;190:381-397.

29. Dyakonov T, Yang CH, Bush D, Gosangari S, Majuru S, Fatmi A. Design and characterization of a silk-fibroin-based drug delivery platform using naproxen as a model drug. J Drug Deliv. 2012;2012:490514.

30. Kawabata M, Imamura T, Miyazono K. Signal transduction by bone morphogenetic proteins. Cytokine Growth Factor Rev. 1998;9(1):49-61.

31. Wang EA, Rosen V, Cordes $P$, et al. Purification and characterization of other distinct bone-inducing factors. Proc Natl Acad Sci US A. 1998;85: 9484-9488.

32. Lynch MM, Liu J, Nigra M, Coppens MO. Chaperonin-inspired $\mathrm{pH}$ protection by mesoporous silica SBA-15 on myoglobin and lysozyme. Langmuir. 2016;32(37):9604-9610.

33. Citak A, Erdem B, Erdem S, Öksüzoğlu RM. Synthesis, characterization and catalytic behavior of functionalized mesoporous SBA-15 with various organo-silanes. J Colloid Interface Sci. 2012;369(1):160-163.

34. Xie W, Hu L, Yang X. Basic ionic liquid supported on mesoporous SBA-15 silica as an efficient heterogeneous catalyst for biodiesel production. Ind Eng Chem Res. 2015;54(5):1505-1512.

35. Choi Y, Lee JE, Lee JH, Jeong JH, Kim J. A biodegradation study of SBA-15 microparticles in simulated body fluid and in vivo. Langmuir. 2015;31(23):6457-6462.

36. Ahmad Nor Y, Niu Y, Karmakar S, et al. Shaping nanoparticles with hydrophilic compositions and hydrophobic properties as nanocarriers for antibiotic delivery. ACS Cent Sci. 2015;1(6):328-334.

37. Song Y, Li Y, Xu Q, Liu Z. Mesoporous silica nanoparticles for stimuliresponsive controlled drug delivery: advances, challenges, and outlook. Int J Nanomedicine. 2016;12:87-110. 
38. Xia L, Lin K, Jiang X, et al. Effect of nano-structured bioceramic surface on osteogenic differentiation of adipose derived stem cells. Biomaterials. 2014;35(30):8514-8527.

39. Reffitt DM, Ogston N, Jugdaohsingh R, et al. Orthosilicic acid stimulates collagen type 1 synthesis and osteoblastic differentiation in human osteoblast-like cells in vitro. Bone. 2003;32(2):127-135.
40. Jiang X, Zhao J, Wang S, et al. Mandibular repair in rats with premineralized silk scaffolds and BMP-2-modified bMSCs. Biomaterials. 2009;30(27):4522-4532.

41. Shah NJ, Macdonald ML, Beben YM, Padera RF, Samuel RE, Hammond PT. Tunable dual growth factor delivery from polyelectrolyte multilayer films. Biomaterials. 2011;32(26):6183-6193.

\section{Publish your work in this journal}

The International Journal of Nanomedicine is an international, peerreviewed journal focusing on the application of nanotechnology in diagnostics, therapeutics, and drug delivery systems throughout the biomedical field. This journal is indexed on PubMed Central, MedLine, CAS, SciSearch $®$, Current Contents $® /$ Clinical Medicine,
Journal Citation Reports/Science Edition, EMBase, Scopus and the Elsevier Bibliographic databases. The manuscript management system is completely online and includes a very quick and fair peer-review system, which is all easy to use. Visit http://www.dovepress.com/ testimonials.php to read real quotes from published authors.

Submit your manuscript here: http://www.dovepress.com/international-journal-of-nanomedicine-journal 